\title{
Design and implementation of photo printer system based on wechat public platform
}

\author{
Li Fan \\ Beijing University of Posts and Telecommunications \\ Beijing, China \\ lifan@bupt.edu.cn
}

\begin{abstract}
This paper introduces the design and implementation of a self-service photo printing system based on wechat public platform. The users can upload photos through wechat public platform, edit photo and select size through the web, then print photo using the machine. Mobile client is developed based on wechat public platform. Server is based on Linux system, and the system of the machine is Windows. The main developing language is python.
\end{abstract}

Keywords: Photo Printer; Wechat Public Platform; Python

\section{INTRODUCTION}

In recent years, with the rapid development of mobile Internet, smart hardware is also becoming more productive. Mobile operating systems iOS and Android occupied the most share of the market. Therefore, App development is almost developed on both systems. However, the technology on two systems is so different and it will increase the difficulties in order to satisfy needs of both iOS and Android users. So there are some platform-level super apps, wechat is one of them. For not very complex needs, wechat public platform provides a unified solution. The way developed on wechat public platform not only decreases the costs, but also provides a powerful flow of support.

This paper introduces the basic principles and processbased wechat public platform for self-printing devices work, then describe the implement of server and clients. Finally, we will show the actual device prototyping.

\section{SYSTEM OVERVIEW}

The system consists of two parts---hardware and software. The software part includes printer terminal client and web server. The hardware part is the printing machine. The server is mainly responsible for connecting wechat public platform and receive, store, process photos. It will receive print information sent by user, and communicate with the client software. The client software is mainly responsible for downloading photos from server, as well as displaying and processing photos. It will place orders to the server and receive payment notification, then print the photos. The basic flow chart is as follows:

\author{
You Xiangdong \\ Beijing University of Posts and Telecommunications \\ Beijing, China \\ youxiangdong@bupt.edu.cn
}

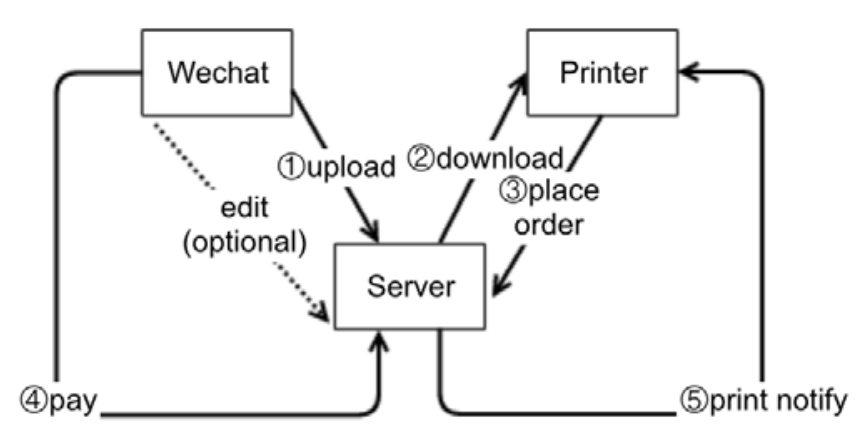

Figure 1: structure of system

\section{A. Wechat Public Platform}

Mobile client development is based on wechat public platform. Users can communicate with server on dialogue window within the wechat public platform and web page in wechat browser. Wechat public platform provides a series of simple formats to transfer data, including text, pictures, videos, music, links, location and other types. Users can send photo directly to the public platform and wechat server will send it to the specified server according to the settings in wechat admin site, with $\mathrm{xml}$ format. The http server receives the message and then extracts information from xml string. Finally, the server will pack the message which we want to response with $\mathrm{xml}$ format. An interaction is completed so. This process is similar to the form of $\mathrm{C} / \mathrm{S}$ structure, using the http protocol based on the wechat public platform.

In addition to the request - response form, users can also receive notification messages from the wechat public platform, which is convenient to the long-time tasks (such as image processing, waiting for the result of payment response, etc.)

As a very popular social app, the wechat has so many users. In traditional application, users need to register an account to get the app's service, the app server can track use by the account. But this will be more convenient on wechat public platform, because it provides the user's unique identify to our server, which can track the target user. Users have no need to register new account any more.

The interactive dialog box of wechat public platform also has its limitations. It only supports some simple formats, and the process flow is so simple. For more complex operations we 
can create web site for users to visit in wechat web browser, which can communicate directly and freely.

\section{B. Server}

The web server is the central part of the whole system. It is responsible for storing data, reading and processing photos and operating database. The server program receives and resolves messages forwarded by wechat public platform. It will download photo from wechat server by the information sent by wechat, and then handle it and response to the wechat text message. As with the action in web page, it is typical B/S structure, and the server will provide web pages for users to display and manipulate data. For the software program of printer, functions such as downloading photos, paying and managing orders are needed. So as the bridge of printer client and wechat platform form, the server should have standardized interface and proper design of database.

The database is response for store all data, including user information, photos, orders, printer information and so on. There should be different modules to process the request.

\section{Clients}

The printer client software is response for extracting and previewing photos, placing orders to the server, as well as managing printers. Every picture is related to unique extraction code, which is generated and returned from the wechat public platform. The extraction code should be valid only within a certain period of time. The client presents photo to the user after being downloaded by correct code. When user confirm that photo is ok, the client will request to the server and place order. in order to ensure the security of payment, all orders should be placed through the web server, client software does not send payment request to a third party directly. After placing order, the client will wait for the result of payment of placed order, then print photos according to the result of payment. This is how the client works.

Also, for the reason of security, the communication between clients and server should have way to check signature to ensure that request is not fake. We use symmetric-key algorithm. The client and server has the same secret key and client encrypts http request parameters. Server encrypt it using same key and compare the result to signature. This can ensure the reality of request.

\section{IMPLEMENTATION}

\section{A. Wechat and Linux Server}

First, building the http server which can be visited in public network and the wechat server will forward message to it according to the setting in wechat public platform admin pages. As wechat development document said, the callback url and secret token add needed. Also, a secret key should be given when encrypt and decrypt messages.

The server should response for different message which sent by wechat server in different formats, including the text messages, picture messages and menu messages. According to the user's instructions, server specifies corresponding response rules. After receiving picture messages, server will download and store the image, as well as processing operations such as cropping, resizing, compressing photos and so on. Then server gives the notification to the user with the unique extraction code. Menu message contains functions such as showing helpful tips, viewing order history.

The size ratio of photos which users upload is not fixed. To make printed photo have good look, the server provides user two ways to solve it. First one, the photos are handled automatically by the system, without cropping the original images. White borders are added to it to ensure consistent ratio. Secondly, users crop it in web page manually. In this way the server needs to provide pages for cropping and previewing. This function is implemented within the wechat browser. Also, the browser web page can also provide more extensions.

The Server program is developed with django ${ }^{[2]}$, a popular web framework of python. The module completing handling image is developed with PIL ${ }^{[3]}$, a kind of image toolkit written by python with friendly interface. Users communicate with the wechat public number as follows:

1) Users focus the wechat public number and send photos, the server will response photo editing links and extraction code to user.

2) If user has no need to edit photos, to Step 4 directly;

3) Click the link to jump to the wechat web page, select the print size and press ok button. After the crop action of server is finished, user can preview crop result in new page, and the user will also get the notification of complete from the server.

4) Download photo by the extracted code in printer client.

\section{B. Terminal Client System}

The client is responsible for extracting, displaying photos, placing orders and receiving payment result and printing photos.

Users communicate with the device as follows:

1) In the main page of client software, user can enter the code, which is received from wechat public platform. This will download the corresponding photo and preview it. The extraction code is valid within a period of time.

2) There are some optional functions, including of rotating pictures, adding borders to the picture, selecting the number of printing and so on. Go to the next when user have no doubt on this step.

3) Select the way of payment, including the Beijing public transportation card payments and wechat payment. When using wechat payment, user need to open camera in wechat app and scan the QR code, then pay for the photo following the instructions.

4) When client receives payment notice successfully, photo printing software will print photo and users should wait until it completes.

When using wechat payment, the server program needs to cooperate with wechat server and terminal client. The notification is based on web-socket protocol, which is a 
communication method of full duplex, not like http, which is half duplex. The server can send message actively to clients and can avoid frequent requests, which is wasting bandwidth.

Card payment needs the dynamic link library interface provided by card manufacture. Interfaces include active, inquiry, payment and so on.

\section{Printers Admin}

In order to manage many printer machines, the server system provides visual web interface to operate database. Those features are:

1) Receive period heartbeat packet from clients to ensure that every machine is online and can work.

2) When there are some problems on clients, such as offline, lacking of print paper, hardware problem. Administers can receive notifications from server in some way automatically (email, SMS, wechat message).

3) Detect and record online and offline logs of machine.
4) Manage clients remotely. Manage the admin password online in dynamic.

5) Manage users, orders, uploaded photos, provide user feedback and other functions.

\section{CONCLUSION}

This paper design and implement a photo printer system. The system is self-service and support multipliable size, including 1 inch, 2 inch, 6 inch, 8 inch etc. User can get real photo immediately by uploading photo to wechat public platform and get it in client software and print.

[1] Deshen Z, Hongliang Q. WeChat Public Platform DevelopmentSubscription Function Research[J]. Electronic Technology \& Software Engineering, 2013, 19: 66-68.

[2] Forcier J, Bissex P, Chun W. Python web development with Django[M]. Addison-Wesley Professional, 2008.

[3] Lundh F, Ellis M. Python imaging library (PIL)[J]. 2012.

[4] Summerfield M. Rapid GUI programming with Python and Qt: the definitive guide to PyQt programming[M]. Pearson Education, 2007. 